CARNETS DE Carnets de géographes

GÉOGRAPHES.

12 | 2019

Géographie(s) des mobilisations

\title{
The Cables and the Power
}

Mobilising Space, Mobilising for Space in the Palestinian refugee camps of Beirut (2014-2017)

\section{Alex Mahoudeau}

\section{OpenEdition}

Journals

Electronic version

URL: http://journals.openedition.org/cdg/5066

DOI: $10.4000 /$ cdg.5066

ISSN: $2107-7266$

Publisher

UMR 245 - CESSMA

Electronic reference

Alex Mahoudeau, "The Cables and the Power », Carnets de géographes [Online], 12 | 2019, Online since 30 December 2019, connection on 24 September 2020. URL : http://journals.openedition.org/cdg/ 5066 ; DOI : https://doi.org/10.4000/cdg.5066

This text was automatically generated on 24 September 2020

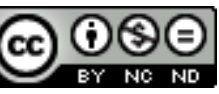

La revue Carnets de géographes est mise à disposition selon les termes de la Licence Creative Commons Attribution - Pas d'Utilisation Commerciale - Pas de Modification 4.0 International. 


\title{
The Cables and the Power
}

\author{
Mobilising Space, Mobilising for Space in the Palestinian refugee camps \\ of Beirut (2014-2017)
}

\section{Alex Mahoudeau}

1 L'ambition de cette thèse est d'explorer la dimension spatiale des mobilisations dans les camps de réfugiés palestiniens de Beyrouth. Situés aux marges (géographiques et sociales) des grandes villes libanaises depuis leur constitution à la fin des années 1940, les camps de réfugiés ont fait l'objet de très nombreuses études, particulièrement depuis les années 1970. Dans la période de l'après-guerre civile libanaise, les camps et leurs habitant.e.s sont l'objet d'un phénomène de relégation sociale, économique, et politique, mis en lumière par Julie Peteet (2005) et Daniel Meier (2008). Ce processus s'illustre également par la dégradation matérielle des camps, objets d'un urbanisme informel et d'un manque de reconstruction systématique après la guerre (figure 1). Dans le même temps, on a observé un phénomène d'« ONG-isation » du gouvernement des camps, dans lequel des associations locales et internationales, formelles ou non, prennent en charge une part importante de l'action sociale dans les camps. L'importance croissante de l'économie informelle dans l'accès à l'eau et à l'électricité a également contribué sur la période à changer le mode de gouvernement des camps, qui apparaît de plus en plus complexe aux yeux des habitant.e.s durant les années $2000 \mathrm{du}$ fait qu'une augmentation du nombre d'acteurs engagés.

2 La façon dont les habitant.e.s des camps se représentent le politique et interagissent avec ce domaine de la vie sociale a été posée de façon répétée depuis le début des années 2000 (Allan, 2014; Hanafi et Long, 2010; Kortam, 2011; Sayigh, 2011). L'apparente désertion du secteur partisan par les habitant.e.s des camps et le développement de mobilisations autour d'associations et sur des enjeux portant sur l'intégration à la société libanaise, notamment le droit au travail, et de problèmes urbains, notamment l'accès aux infrastructures et la reconstruction, ont généré un débat sur la « dépolitisation » dans les camps de réfugiés.

3 Dans cette thèse, je suis principalement trois réseaux d'activistes ${ }^{1}$ au sein des trois camps de Beyrouth (Chatila, Mar Elias, et Burj el-Barajneh). Ces réseaux ont été analysés à travers une enquête de terrain qualitative de neuf mois prêtant attention 
aux modes d'actions, carrières, formes de l'interaction en public, représentations politiques, et rapport à l'espace des activistes, approfondie par des entretiens avec des acteurs-clé (Comités Populaires, organismes de financement, UNRWA²). Je prends ainsi le même parti que plusieurs travaux récents, qui décrivent la façon dont les engagements tournés vers le «local» comme des réinvestissements sous d'autres formes d'engagements politiques (Abou Zaki, 2016; Allan, 2014; Hassan et Hanafi, 2010). En prêtant attention au rapport des activistes à l'espace des camps ainsi qu'au fonctionnement au quotidien de leurs groupes, je montre que l'intérêt pour les infrastructures, décrites comme «locales ", procède moins d'une "dépolitisation » de ces acteurs que du réinvestissement de ressources sociales selon des grammaires différentes. Elle propose à ce titre une jonction théorique entre sociologie pragmatique et géographie sociale concernant l'étude des mouvements sociaux.

4 En effet, ainsi que l'illustre le premier chapitre, l'espace des camps, tout comme ses modes de gestion, ne peut être décrit de façon univoque. À travers l'analyse du paysage et de sa perception par les activistes, l'investissement mémoriel et identitaire décrit par une partie de la littérature se trouve accompagné d'autres formes de représentations. Le camp apparaît tout à la fois comme lieu dépositaire d'une identité collective palestinienne en exil, de rapports différenciés au nationalisme, d'activités économiques, culturelles, sociales, mais aussi d'une inscription dans la ville informelle et pauvre beyrouthine. C'est en mobilisant ces divers aspects de l'expérience « locale » qui transparaissent aussi bien dans le matériel que dans les institutions et les représentations, que les activistes produisent à la fois des processus cognitifs de production de représentations ou de discours (ou cadrages) leur permettant de naviguer dans cet espace au quotidien et d'aborder, dans des interactions en public, divers problèmes sociaux. J'identifie trois de ces grammaires, que je qualifie respectivement de partisane, associationnelle, et familière.

5 Les deuxième et troisième chapitres analysent respectivement la constitution d'organisations et de problème publics dans le registre de la grammaire associationnelle, dominante dans les organisations étudiées. Celles-ci se structurent sous forme d'ONG ou d'associations, moins sous l'effet d'une conversion idéologique de leurs membres que de transformations dans les conditions d'accès aux financements durant et après les dernières années de guerre civile. Les activistes disposant des compétences techniques et d'une capacité à se représenter le monde dans les termes des ONG réussissent alors à imposer ces représentations aux organisations dont ils et elles font partie. Le troisième chapitre montre la façon dont, pendant mon enquête de terrain, les activistes emploient la grammaire associationnelle et les modes d'action qui lui sont associés (enquête, prise en charge directe des problèmes, notamment) pour produire des problèmes locaux, les "problèmes du camp ", qui leur permettent de dénoncer les partis et les Comités Populaires qui en émanent, tout en évitant d'être étiquetés comme "politiques» par les habitant.e.s des camps. En parvenant à cadrer l'expérience quotidienne de l'espace des camps comme la preuve d'un échec de la part de ces acteurs " politiques », les activistes parviennent ainsi par étapes à les dénoncer publiquement. Cette dénonciation apparaît particulièrement dans une série de débats publics organisés par les activistes en 2015-2016 et aboutissant à un appel à une transformation radicale de l'organisation du gouvernement des camps.

6 Le quatrième chapitre se concentre sur la façon dont cette séquence d'événements, si elle n'a pas conduit aux résultats attendus par les activistes, a eu des effets rétroactifs 
sur l'espace des camps en lui-même : par leurs actions, les groupes suivis ont influencé la réalité matérielle et symbolique des camps (notamment à travers la toponymie ayant cours dans ces derniers). Ils ont également reconfiguré la dimension scalaire des camps, en déplaçant des conflits et problèmes strictement «locaux » à une échelle «translocale ». Enfin, je montre que la production de l'espace est en partie influencée par ces actions collectives, notamment à travers la manière dont les activistes « ancrent » leurs représentations collectives dans l'espace, y compris matériel.

Figure 1 : Burj al-Barajneh, Beyrouth, discours nationaliste, infrastructures informelles, et dégradation du bâti

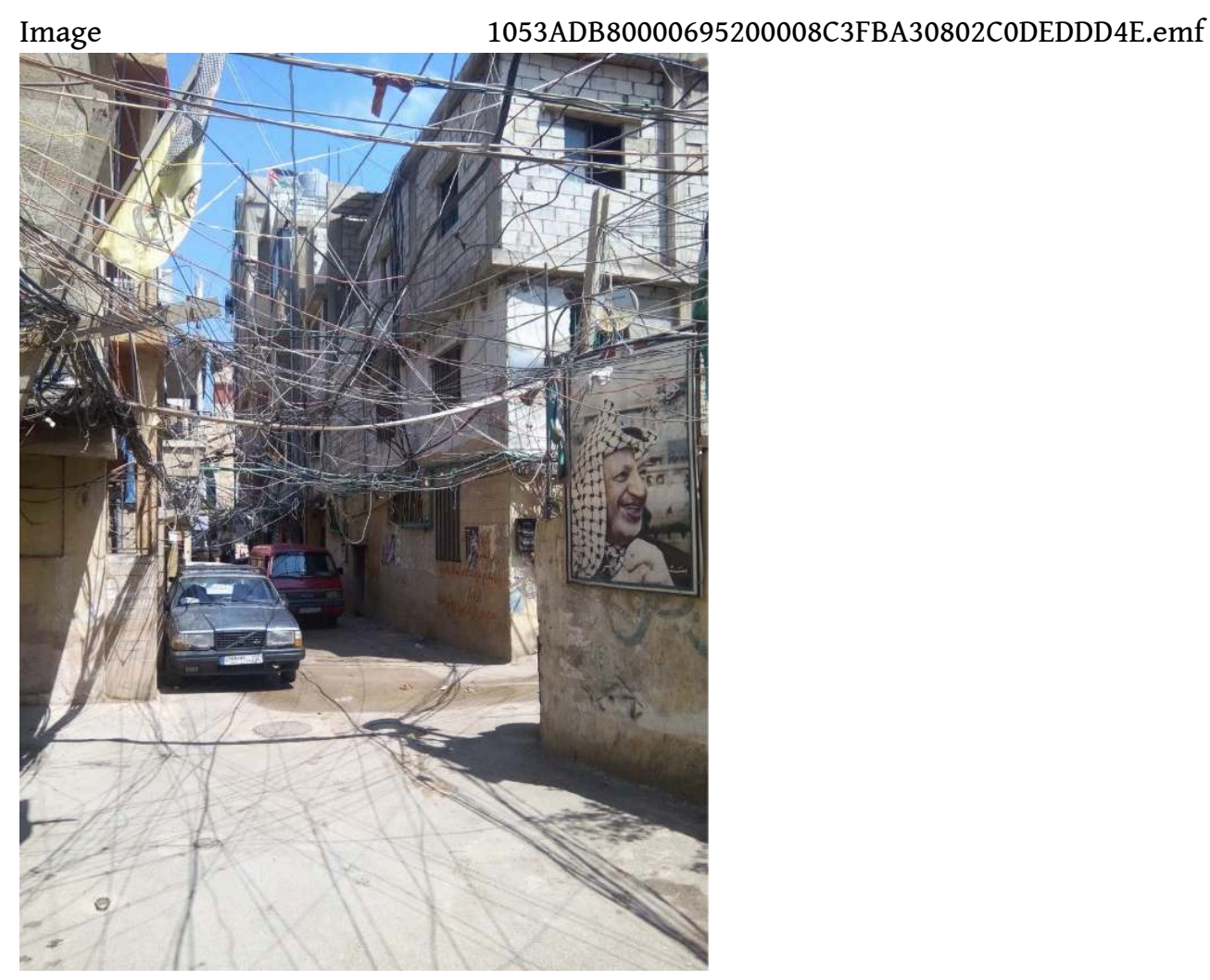

Lien électronique si la thèse est disponible en ligne

https://halshs.archives-ouvertes.fr/tel-01786341

\section{Discipline}

Science Politique

\section{Directeur}

Jeroen Gunning

\section{Université}

King's College London

Membres du jury de thèse, soutenue le 23 novembre 2017 :

Laleh KHALILI, (membre Interne, School of Oriental and Arab Studies)

Fabrice RIPOLL (membre externe, Maître de conférences, Université Paris-Est

Créteil) 
Situation professionnelle à l'issue de la thèse

Post-doctorant, Groupe Transversal Justice, Espace, Discriminations, Inégalités,

Labex « Futurs Urbains », Université Paris-Est (septembre 2018-septembre 2020)

Courriel de l'auteur

mahoudeau.alex [at] gmail.com

\section{BIBLIOGRAPHY}

ABOU ZAKI H.c. (2016), « Repenser le politique dans le camp de Chatila : l'expérience des Ahali », A contrario, $\mathrm{n}^{\circ} 23$, pp. 57-75.

ALLAN D. (2014), Refugees of the revolution: experiences of Palestinian exile, Stanford, California, Stanford University Press (Stanford studies in Middle Eastern and Islamic societies and cultures), $309 \mathrm{p}$.

HANAFI s., LONG T. (2010), « Governance, Governmentalities, and the State of Exception in the Palestinian Refugee Camps of Lebanon ", Journal of Refugee Studies, vol. 23, n², pp. 134-159.

HASSAN I.S., HANAFI s. (2010), « (In)Security and Reconstruction in Post-conflict Nahr al-Barid Refugee Camp », Journal of Palestine Studies, vol. 40, n¹, pp. 27-48.

KORTAM M. (2011), « Politics, Patronage and Popular Committees in the Shatila Refugee Camp, Lebanon », dans HANAFI S., KNUDSEN A.J. (dirs.), Palestinian refugees : identity, space and place in the Levant, London, New York, Routledge, pp. 193-204.

MEIER D. (2008), Mariages et identité nationale au Liban : les relations libano-palestiniennes dans le Liban de Taëf, 1989-2005, Genève, Paris, Institut de hautes études internationales et du développement, Karthala, 216 p.

PETEET J. (2005), Landscape of hope and despair Palestinian refugee camps, Philadelphia, Pa., University of Pennsylvania Press, $280 \mathrm{p}$.

SAYIGH R. (2011), « Palestinian Camp Refugee Identifications: a New Look at the "Local" and the "National" » in Hanafi s., Knudsen A. J. (dirs.), Palestinian refugees : identity, space and place in the Levant, London, New York, Routledge, pp. 50-64.

\section{NOTES}

1. Ce terme renvoyant dans la bouche des acteurs aussi bien à des activités militantes qu'à la participation bénévole ou non à des activités associatives.

2. Office de Secours des Nations Unies pour les Réfugiés Palestiniens au Proche-Orient 


\section{AUTHOR}

\section{ALEX MAHOUDEAU}

Docteur en science politique, post-doctorant au groupe JEDI (Justice, Espace, Discriminations, Inégalités) du Labex « Futurs urbains » alex.mahoudeau[@]u-pem.fr 